surfactant, varespladib, both or nothing (overall $p=0.016$; Dunnett post-hoc between cultures treated with varespladib and varespladib+surfactant against untreated cultures $p=0.01$ ). FFA are higher in untreated cultures $(394 \pm 82 \mu \mathrm{M})$, than in surfactant$(219 \pm 70 \mu \mathrm{M})$ and in varespladib-treated ones $(148 \pm 51 \mu \mathrm{M})$. Combined treatment reduced FFA to $206 \pm 47 \mu \mathrm{M}$ (overall $\mathrm{p}=0.017$; Sidak post-hoc $p=0.036$ and $p=0.023$ for the varespladib and combined treatment against control cultures).

Conclusions The joined administration of varespladib and poractant- $\alpha$ significantly reduce sPLA2 activity and FFA production. Surfactant+varespladib affect sPLA2 pathway significantly more than the surfactant alone.

\section{THE PEDIATRIC ALIEN STUDY: INCIDENCE AND OUTCOME OF THE ACUTE RESPIRATORY DISTRESS SYNDROME IN CHILDREN}

doi:10.1136/archdischild-2012-302724.0059

${ }^{1} Y$ Lopez, ${ }^{2} \mathrm{~A}$ Martinez de Azagra, ${ }^{3} \mathrm{~A}$ Medina, ${ }^{4} \mathrm{~A}$ Rodriguez, ${ }^{5} \mathrm{D}$ Arjona, ${ }^{6} \mathrm{E}$ Alvarez, ${ }^{7}$ E Oñate, ${ }^{8} \mathrm{~J}$ Villar, PEDALIEN Network. 'Universitary Hospital Cruces, Baracaldo; ${ }^{2} \mathrm{HU}$ Niño Jesus, Madrid; ${ }^{3} \mathrm{HUC}$ Asturias, Oviedo; " $H U X$ Santiago, Santiago de Compostela; ${ }^{5} \mathrm{H}$ Virgen de la Salud, Toledo; ${ }^{6} \mathrm{H}$ Ramon y Cajal, Madrid; ' Complejo Hospitalario Donostia, Donostia; ${ }^{8 H U}$ Juan Negrin, Las Palmas, Spain

Introduction The incidence and outcome of the acute respiratory distress syndrome (ARDS) in children is not well known, especially under current ventilatory practices. The goal of this study was to determine the incidence, etiology and outcome of ARDS in the pediatric population in the setting of lung protective ventilation.

Method A 1-year, prospective, multicenter, observational study in 12 geographical areas of Spain covered by 21 pediatric intensive care units (PICUs).

Results Data on ventilatory management, gas-exchange, hemodynamics, and organ dysfunction were collected. A total of 146 mechanically ventilated patients fulfilled the ARDS definition, representing an incidence of 3.9/100,000 population $\leq 15$ years of age/year. Pneumonia, sepsis and respiratory syncytial virus-related infection were the most common causes of ARDS. At the time of meeting ARDS criteria, mean $\mathrm{PaO}_{2} / \mathrm{FiO}_{2}$ was $99 \pm 41 \mathrm{mmHg}$, mean tidal volume was $7.6 \pm 1.8 \mathrm{ml} / \mathrm{kg}$ predicted body weight, mean plateau pressure was $27 \pm 6 \mathrm{cmH}_{2} \mathrm{O}$, and mean PEEP was $8.9 \pm 2.9 \mathrm{cmH}_{2} \mathrm{O}$. Overall ARDS PICU and hospital mortality was $26 \%$ (95\%CI: 19.6-33.7) and 27.4\% (95\%CI: 20.8-35.1), respectively. At 24 h, after assessment of oxygenation under standard ventilatory settings, 118 (80.8\%) patients continued to meet ARDS criteria $\left(\mathrm{PaO}_{2} / \mathrm{FiO}_{2} 104 \pm 36\right.$ mmHg; PICU mortality $30.5 \%$ ) whereas 28 patients (19.2\%) had a $\mathrm{PaO}_{2} / \mathrm{FiO}_{2}>200 \mathrm{mmHg}$ (PICU mortality 7.1\%) ( $\mathrm{p}=0.014$ ).

Conclusions This is the largest study to estimate prospectively the pediatric population-based ARDS incidence and the first incidence study performed during the routine application of lung protective ventilation in children. Our findings support a lower ARDS incidence and mortality than those reported for adults.

\section{COMPARISON BETWEEN AEROSOLIZED PERFLUOROCARBON AND PARTIAL LIQUID VENTILATION IN PRETERM LAMBS WITH SEVERE RESPIRATORY DISTRESS SYNDROME}

doi:10.1136/archdischild-2012-302724.0060

'X Murgia, ${ }^{2} \mathrm{~A}$ Valls-i-Soler, 'V Mielgo, 'E Ruiz-del-Yerro, 'E Goikoetxea, ' ${ }^{3} \mathrm{~L}$ Andrés, ${ }^{3} \mathrm{~L}$ Marín-Cano, ${ }^{4} \mathrm{~S}$ Gómez-Urquijo, ${ }^{1} \mathrm{C}$ Rey-Santano. ${ }^{1}$ Research Unit; ${ }^{2}$ Neonatal Intensive Care Unit; ${ }^{3}$ Department of Pathology, Cruces University Hospital, Barakaldo; 'Department of Neurosciences, University of the Basque Country, Leioa, Spain

Background and aim Perfluorocarbon (PFC) aerosolization is feasible; however, it is unknown whether aerosolization is better than Partial Liquid Ventilation (PLV).
Methods 18 preterm lambs were randomly assigned to receive aerosolized PFC $(10 \mathrm{ml} / \mathrm{kg} / \mathrm{h}$ for $2 \mathrm{~h})$ delivered via an inhalation catheter, (PFC-aero group), instilled intratracheal PFC $(20 \mathrm{ml} / \mathrm{kg}$; PLV group), or just mechanical ventilation (CONTROL group). Gas exchange, pulmonary mechanics, and histological scores were assessed. Mean \pm SD, ANOVA, $p<0.05$.

Results Both PFC administration techniques significantly improved gas exchange and pulmonary mechanics compared to CONTROL group (two-way ANOVA). 15 minutes after PLV, OI and VEI were significantly better in the PLV group compared to other groups. However, in terms of OI, aerosolized PFC remained significantly better than CONTROL group for the entire observational period (360 $\mathrm{min}$ ), whereas at $240 \mathrm{~min}$ and on, the differences between PLV and CONTROL groups were not significant. PLV and aerosolized PFC significantly decreased the degree of atelectasis but did not significantly improve the general histological score.

\section{Abstract 60 Table 1}

\begin{tabular}{lllll}
\hline $\begin{array}{l}\text { OXYGENATION } \\
\text { INDEX }\end{array}$ & BASELINE & $\mathbf{1 h}$ & $\mathbf{3 h}$ & $\mathbf{6 h}$ \\
\hline Control & $79(52)$ & $46(18)$ & $52(30)$ & $64(27)$ \\
PLV & $102(49)$ & $6(1)^{*} \#$ & $12(6)^{*}$ & $32(35)$ \\
PFC-aero & $71(45)$ & $23(11)^{*}$ & $10(2)^{*}$ & $18(18)^{*}$ \\
\hline
\end{tabular}

${ }^{*}$ vs. CONTROL; \# vs. PFC-aero. One-way ANOVA

Conclusion Both PFC administration techniques show pulmonary efficacy in RDS. Future research should focus on the PFC aerosol delivery efficiency.

\section{ASSOCIATION OF VITAMIN D RECEPTOR GENE POLYMORPHISMS AND BRONCHOPULMONARY DYSPLASIA}

doi:10.1136/archdischild-2012-302724.0061

${ }^{1} \mathrm{O}$ Altun Koroglu, ${ }^{2} \mathrm{H}$ Onay, ${ }^{1} \mathrm{~B}$ Cakmak, 'B Bilgin, ${ }^{1} \mathrm{M}$ Yalaz, ${ }^{2} \mathrm{~S}$ Tunc, ${ }^{2} \mathrm{~F}$ Ozkinay, ${ }^{1} \mathrm{~N}$ Kultursay. ${ }^{1}$ Neonatology; ${ }^{2}$ Medical Genetics, Ege University Faculty of Medicine, Izmir, Turkey

Background and aims Vitamin D is considered as an important regulator of fetal lung development and innate immune system. Its functions involved in susceptibility and resistance to infections and pulmonary diseases may be important for the occurrence of bronchopulmonary dysplasia (BPD). The aim of the study was to investigate the relationship between Vitamin D receptor gene polymorphism and BPD in preterm infants.

Methods Fok I, Bsm I, Apa I, and Taq I polymorphisms in the Vitamin D Receptor (VDR) gene were genotyped using restriction fragment length polymorphism in 109 preterm infants (47 with BPD, 62 without BPD) born at gestational age $\leq 32$ weeks and admitted to NICU at Ege University Hospital.

Results The univariate analysis showed Ff $(\mathrm{OR}=3.937, \mathrm{p}=0.022$, $95 \% \mathrm{CI}=1.22-12.69)$ and $\mathrm{ff}(\mathrm{OR}=5.238, \mathrm{p}=0.004,95 \% \mathrm{CI}=1.69$ 16.23) genotypes of Fok I polymorphism were associated with increased risk of BPD; whereas tt genotype of Taq 1 polymorphism; was associated with a protective effect against $\mathrm{BPD}(\mathrm{OR}=0.30$ $\mathrm{p}=0.04,95 \% \mathrm{CI}=0.098-0.094)$. In a multivariate logistic regression analysis of the model including variant Fok1 genotype with significant PDA, clinical and culture proven sepsis, mechanical ventilation and surfactant treatment; variant Fok 1 genotype increased the risk of BPD $(\mathrm{OR}=4.115, \mathrm{CI}=1.080-15.686, \mathrm{p}=0.038)$ independent from these factors. Taq 1, Bsm 1 and Apa 1 polymorphisms did not have any effect in the same model.

Conclusion Fok1 polymorphism was associated with increased frequency of BPD after adjusting for multiple confounders. VDR gene polymorphisms may be suitable for prediction of infants at high risk for BPD. 